\title{
Isotope shifts and hyperfine structures investigation of doubly excited levels in SrI
}

\author{
M. Barsanti *, L. Gianfrani, F. Pavone ${ }^{\star}$, A. Sasso, C. Silvestrini, and G.M. Tino \\ Dipartimento di Scienze Fisiche, Universitả di Napoli „Federico I"“, Mostra d'Oltremare Pad. 20, 1-80125 Napoli, Italy
}

Received 22 November 1991; final version 17 January 1992

\begin{abstract}
We measured isotope shifts and hyperfine structure of visible transitions of stable strontium isotopes by means of Doppler-free saturated absorption spectroscopy. In particular, we investigated transitions between excited states where the upper level involves two excited electrons. We report hyperfine coupling constants for the levels $5 \mathrm{p} 4 \mathrm{~d}^{1} \mathrm{D}_{2}, 5 \mathrm{p} 4 \mathrm{~d}^{3} \mathrm{~F}_{2}, 5 \mathrm{~s} 4 \mathrm{~d}^{3} \mathrm{D}_{1}, 5 \mathrm{~s} 6 \mathrm{~s}^{3} \mathrm{~S}_{1}, 5 \mathrm{~s} 5 \mathrm{p}$ ${ }^{3} \mathrm{P}_{1}$ and, for some of the studied transitions, we separate the specific mass and volume contribution to the isotope shifts.
\end{abstract}

PACS: $35.10 . F ; 31.30 . G$

\section{Introduction}

In strontium atom $(Z=38)$, like all alkaline-earth elements, the outer electrons are extremely sensitive to the effects of electron correlation which lead to some influences in the isotope shifts (IS) and hyperfine structure (hfs).

In the last years many experimental and theoretical investigations on IS and hfs of this element were performed [1-3]. While much has been done for transitions involving single excited levels only few studies concern levels originated by doubly excited electrons. Such doubly excited configurations play an important role in the theoretical description of strontium spectrum, because they are the main perturbers of the series. Calculations on level isotope shifts of strontium, expecially in singlet terms, were performed by means of numerical multiconfiguration Hartree-Fock (MCHF) methods [4], in order to study the configuration mixing effects. Measurements on transition isotope shifts [1] are, hence, extremely useful, because they provide a severe test for the validity of these calculations.

\footnotetext{
* Present address: Scuola Normale Superiore, Pisa, Italy $\star \star$ Permanent address: European Laboratory of Nonlinear Spectroscopy, Firenze, Italy
}

In the present paper we report the results of a high resolution laser spectroscopic investigation of several strontium transitions never investigated before. Most of the transitions here studied involve levels where both the optical electrons are excited $\left(5 p^{2}{ }^{1} S_{0}, 5 p^{2}{ }^{1} D_{2}, 5 p 4 d\right.$ ${ }^{1} D_{2}$ and $5 p 4 d^{3} F_{2}$ ). Such levels were reached by laser excitation starting from levels populated by electron impact excitation in a hollow cathode discharge.

Doppler-free spectra were recorded by using mainly saturated absorption spectroscopy that, in this case, resulted more advantageous than other sub-Doppler spectroscopic techniques as, for example, polarization spectroscopy. We measured transition isotope shifts of the four stable isotopes ${ }^{84,86,87,88} \mathrm{Sr}$ and resolved hyperfine structure of ${ }^{87} \mathrm{Sr}$ (nuclear spin $I=9 / 2$ ). Using the values for the mean-square nuclear charge radii $\delta\left\langle r^{2}\right\rangle$ taken from [5], we could separate the two isotope shift contributions, i.e. the specific mass and the volume effect. From the analysis of hyperfine structure splittings we estimate the magnetic dipole and the electric quadrupole coupling constants for several levels of ${ }^{87} \mathrm{Sr}$.

\section{Experimental setup}

A block diagram of the experimental apparatus is shown in Fig. 1. Strontium atoms in the ground and excited states were produced using an argon sustained hollow cathode discharge. The discharge cell was the same of that already used in our previous works [6]; construction details are given in [7]. The discharge was operated at currents of about $75 \mathrm{~mA}$ and was sustained with 1 Torr of argon. The laser source was a single-mode cw ring dye laser (Coherent 699-21) operating with DCM and pumped by an $\mathrm{Ar}^{+}$laser.

Sub-Doppler spectroscopy was performed using a typical experimental arrangement for saturated absorption spectroscopy. The linearly polarized laser beam was split in two parts of different intensities: the pump beam had an intensity of about $10 \mathrm{~mW}$ while the probe beam intensity was about $1 \mathrm{~mW}$. The two counterpropagating 


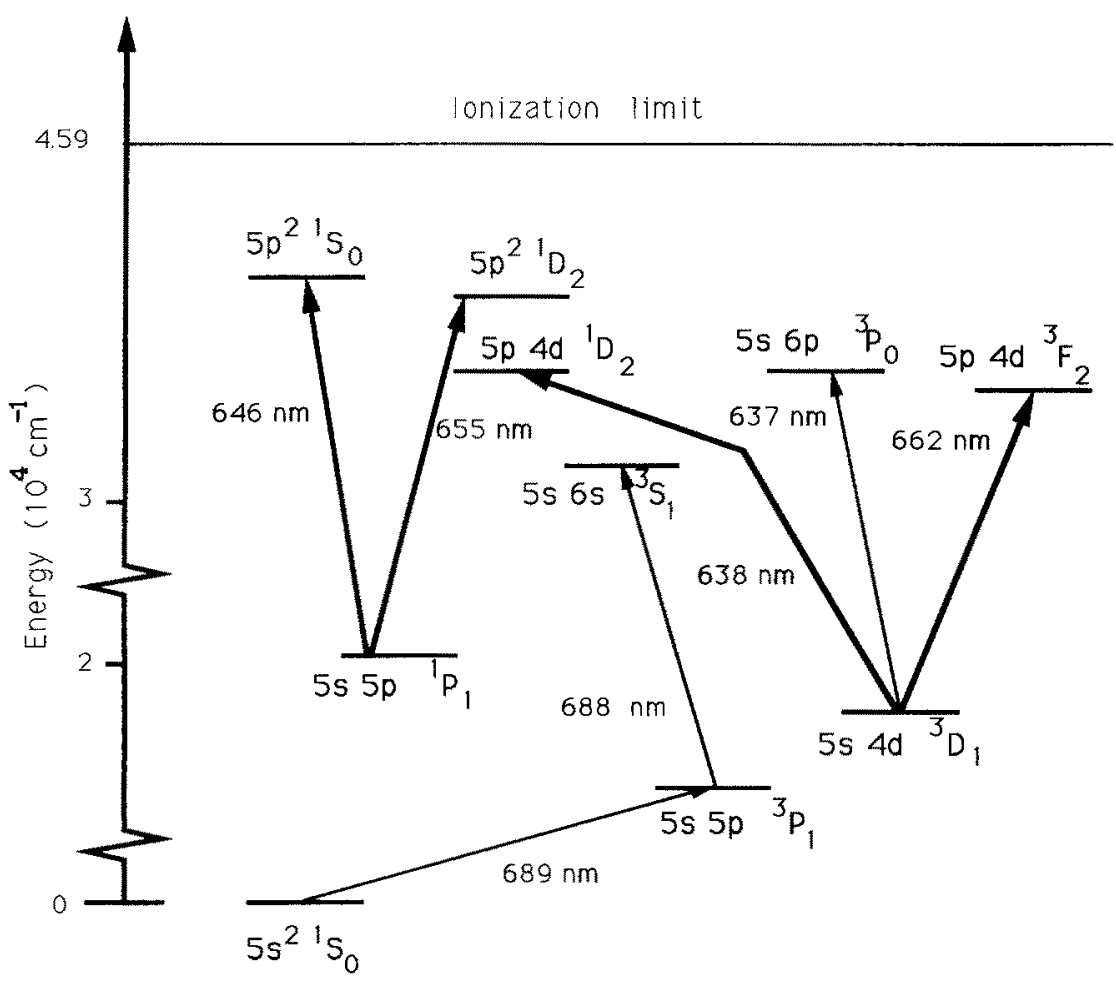

Fig. 1. Relevant levels of strontium atom and transions investigated in this paper. The bold lines represent the transitions involving a doubly excited level beams were superimposed and focused in the hollow cathode bore; a photodiode detected the transmitted intensity of the probe beam.

Laser wavelength was measured using a traveling Michelson interferometer with an accuracy of 1 part in $10^{6}$ while the laser frequency scan was calibrated by means of a high-finesse Fabry-Perot interferometer with a freespectral-range of $75 \mathrm{MHz}$.

\section{Results and discussion}

The relevant energy levels of strontium atom and the transitions investigated in this work are shown in Fig. 2. For some of the transitions reported in Fig. 2 the assignment of hyperfine spectra was made difficult by a simultaneous presence of hfs in both the upper and lower levels and by the occurence of spurious peaks (cross-over signals). In order to simplify such assignments, it can be useful to investigate first a transition in which only one level " $i$ " is affected by hf interaction. The study of transitions with more complicated hf patterns and sharing the level " $i$ " can be then considerably simplified. This is the case, for example, of the transitions $5 s 4 d^{3} D_{1}-5 p 4 d^{1} D_{2} \quad(\lambda=638.0 \mathrm{~nm}) \quad$ and $5 s 4 d^{3} D_{1}$ $-5 p 4 d^{3} F_{2}(\lambda=661.7 \mathrm{~nm})$, whose common lower level is also involved in the $5 s 4 d^{3} D_{1}-5 s 6 p^{3} P_{0}$ $(\lambda=637.0 \mathrm{~nm})$ transition, which exhibits hfs only in the lower ${ }^{3} D_{1}$ level (see Fig. 1). The $5 s 4 d^{3} D_{1}$ splits in three hf components as shown in Fig. $3 \mathrm{a}$. By measuring the hyperfine splittings $\Delta_{11 / 2-9 / 2}$ and $\Delta_{9 / 2-7 / 2}$ the constants $A$ and $B$ can be determined [8]. This allowed us an easier and unambigous identification of the nine peaks which derive from the hfs of the $5 s 4 d^{3} D_{1}-5 p 4 d^{3} F_{2}$ transitions shown in Fig. $3 \mathrm{~b}$. The determination of the best choice of hyperfine constants $A$ and $B$ were accomplished using a standard least-squares method. Using a similar approach the hfs of the ${ }^{3} P_{1}-{ }^{3} S_{1}$ transition $(\lambda=687.8 \mathrm{~nm})$ was also assigned with the help of the intercombination line ${ }^{1} S_{0}-{ }^{3} P_{1}$.

In Table 1 we report the results of hf constants for the levels investigated in this paper and, when available, we compare them with data from other experimental investigations. As can be noted for the $5 s 5 p^{3} P_{1}$ and $5 s 6 s^{3} S_{1}$ levels, we observe a good agreement with experimental values reported in [2]. For the $5 p 4 d^{1} D_{2}$ level the small quadrupole contribution could not be estimated because of the larger error affecting the measured value of $B$. Values of $A$ and $B$ for the ${ }^{1} D_{2}$ level, were more accurately estimated by Grundevik et al. [3] by means of radio-frequency double resonance method and for the constant $A$ we observe a good agreement with our result.

This analysis is also important to calculate the centroid of the $h p$ structure pattern of the odd isotope ${ }^{87} \mathrm{Sr}$ in order to measure the isotope shifts. The isotope shifts of the transitions investigated in this work are reported in Table 2. In the last two columns we also report the ${ }^{86-88} \mathrm{Sr}$ residual isotope shifts and compare them with the theoretical prediction made by Aspect et al. [9], as explained later. Isotope shift values are missing for some isotope couples of the transitions listed in Table 2 for the following reasons. In the case of the $5 s 5 p^{1} P_{1}$ $-5 p^{2}{ }^{1} S_{0}(\lambda=646 \mathrm{~nm})$ transition, as shown in Fig. 4 , the hfs components, and the ${ }^{87} \mathrm{Sr}$ centroid, are completely covered by the most prominent ${ }^{88} \mathrm{Sr}$ peak (homogeneous width $\approx 50 \mathrm{MHz}$ ). This result is consistent with other experimental observations: the hfs of the ${ }^{1} P_{1}$ level was 


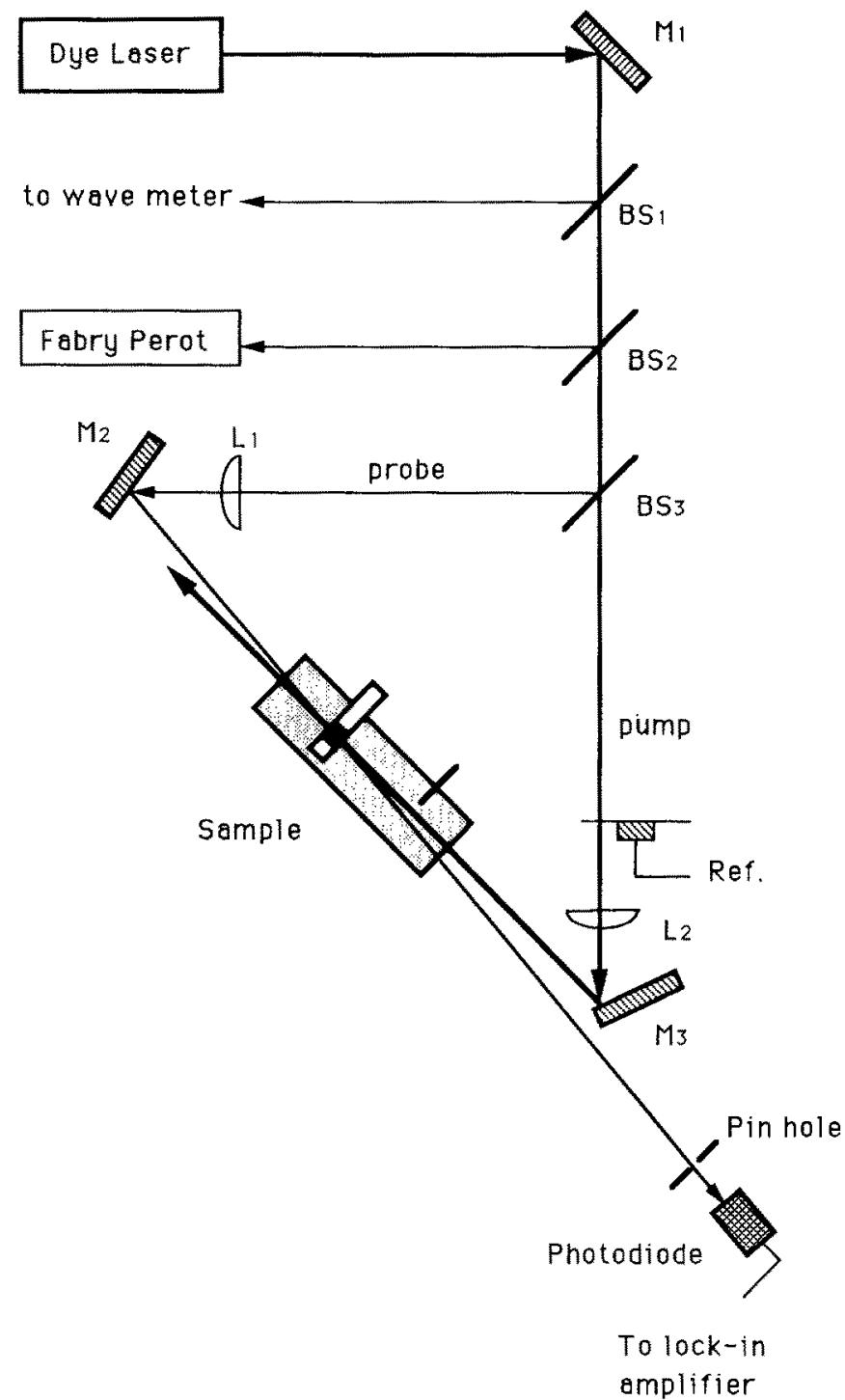

Fig. 2. Schematic experimental apparatus for saturated absorption spectroscopy (BS: beam-splitter; $M$ : mirror; $L$ : lens). Strontium vapors were produced in a hollow cathode discharge
Table 1

\begin{tabular}{llllll}
\hline Level & $A(\mathrm{MHz})$ & & \multicolumn{3}{l}{$B(\mathrm{MHz})$} \\
\cline { 2 - 4 } \cline { 5 - 6 } & $\begin{array}{l}\text { our } \\
\text { results }\end{array}$ & $\begin{array}{l}\text { previous } \\
\text { works }\end{array}$ & & $\begin{array}{l}\text { our } \\
\text { results }\end{array}$ & $\begin{array}{l}\text { previous } \\
\text { works }\end{array}$ \\
\hline $5 s 4 d^{3} D_{1}$ & $140(2)$ & - & $-6(4)$ & - \\
$5 p 4 d d^{3} F_{2}$ & $-50.5(4)$ & - & & $65(3)$ & - \\
$5 p 4 d^{1} D_{2}$ & $-30.0(1.2)$ & $-29.4(3)^{\mathrm{a}}$ & & $-13.6(2)^{\mathrm{a}}$ \\
$5 s 4 p^{3} P_{1}$ & $-262(2)$ & $-260.4(1.1)^{\mathrm{b}}$ & $-37(9)$ & $-35.7(5)^{\mathrm{b}}$ \\
$5 s 6 s^{3} S_{1}$ & $-543(2)$ & $-542.1(4)^{\mathrm{b}}$ & - & - \\
\hline
\end{tabular}

a $[3]$

b $[2]$

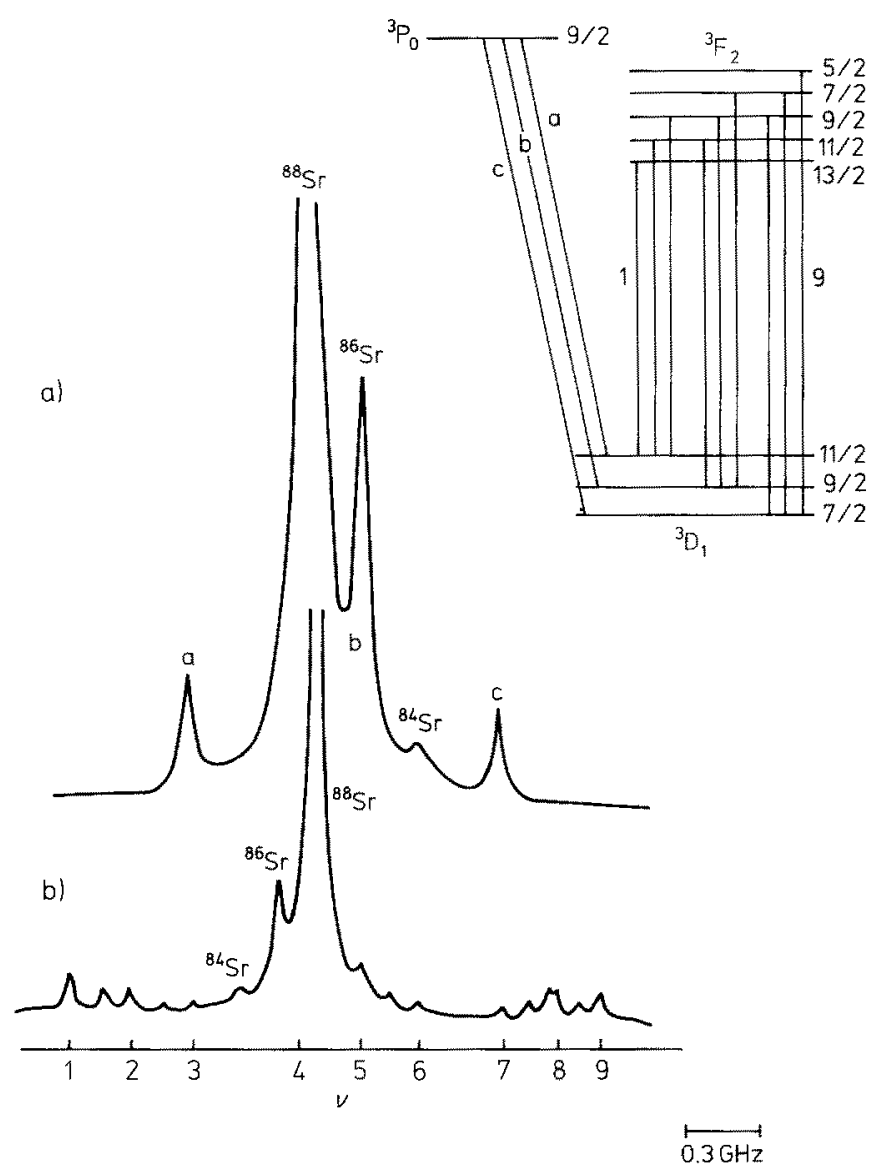

Fig. 3. Saturation spectra of the transitions $5 s 4 d^{3} D_{1}-5 p 6 p^{3} P_{0}$ (part $a$ ) and $5 s 4 d^{3} D_{1}-5 p 4 d^{3} F_{2}$ (part $b$ ). The peak $b$ in $a$ ) is superimposed to the ${ }^{86} \mathrm{Sr}$ peak (Laser power $=10 \mathrm{~mW}$, Ar pressure $=0.6 \mathrm{mbar}$, discharge current $=60 \mathrm{~mA}$ )

Table 2

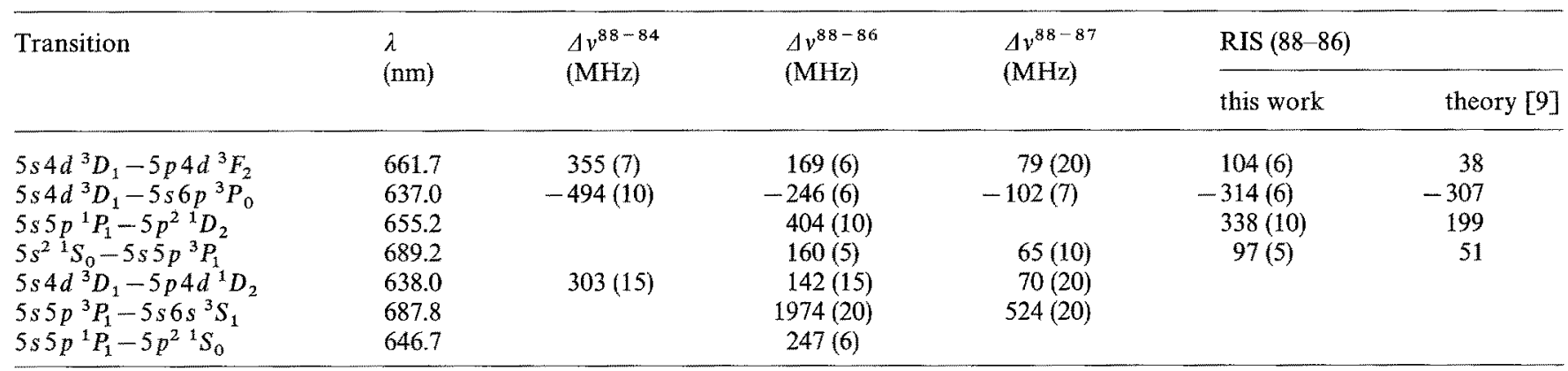


Table 3

\begin{tabular}{lcc}
\hline$\lambda(\mathrm{nm})$ & $\mathrm{F}\left(\mathrm{GHz} / \mathrm{fm}^{2}\right)$ & $\mathrm{M}(\mathrm{GHz})$ \\
\hline 661.7 & $-0.41(0.05)$ & $321(10)$ \\
638.0 & $-0.3(0.2)$ & $240(40)$ \\
637.0 & $0.8(0.6)$ & $-1020(130)$ \\
\hline
\end{tabular}

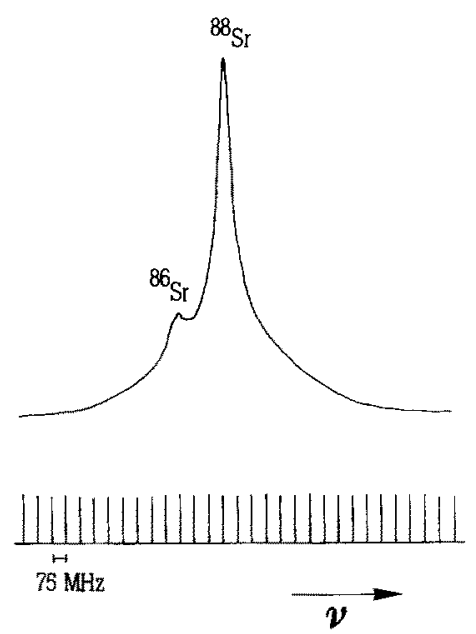

Fig. 4. Doppler free spectra of the $5 s 5 p^{1} P_{1}-5 p^{2}{ }^{1} S_{0}$ transition (Laser power $=10 \mathrm{~mW}$, Ar pressure $=0.7 \mathrm{mbar}$, discharge current$=75 \mathrm{~mA}$ )

also not resolved in [10] being the intervals much less than the measured linewidth of $25 \mathrm{MHz}$. Moreover, from level isotope shift measurements reported in [1] and [10] we can estimate a transition isotope shift ${ }^{87-88} \mathrm{Sr}$ of the $5 s 5 p^{1} P_{1}-5 p^{2}{ }^{1} S_{0}$ line of about $40 \mathrm{MHz}$. The signal-tonoise ratio also limits the detection of rare isotopes as demonstrated in Fig. 4 for the ${ }^{84} \mathrm{Sr}$ isotope (natural abundance $0.56 \%$ ). It is worth to note that the lineshapes shown in Fig. 4 are more affected by a residual Doppler background than those reported in Fig. 3. This is due to a different role played by the velocity-changing collisions for the compared transitions.

The analysis of isotope shifts requires accurate calculation of two shift effects, i.e. volume and specific mass contribution. Such calculations are normally based on multi-configuration Hartree-Fock approaches, which provide the term energies for different isotopes.

Recently, Aspect et al. [9] have calculated by MCHF method residual level isotope shift of ${ }^{86-88} \mathrm{Sr}$ for several levels, referred to the ground state of Sr II. Some of these levels are involved in the transitions we investigated in this work, hence, allow to calculate residual transition isotope shifts and compare them with our experimental values (see last two columns of Table 2). We observe a good agreement only for the transition at $637 \mathrm{~nm}$, while, for the other ones the agreement is quite poor. However, our RIS value on the 689 line agrees with measurements made by Bender et al. [2], while for the other transitions no other experimental results are available.
In order to provide data of more theoretical interest, we separate for the $662 \mathrm{~nm}, 637 \mathrm{~nm}$ and $638 \mathrm{~nm}$ lines the volume contribution to the RIS from the specific mass one. As is well known the coefficients $F$ and $M$ concerning the field and specific mass shift contributions can be estimated by means of King-plots [11]. Including the mean square radii values $\delta\left\langle r^{2}\right\rangle$ from [5], namely

$$
\begin{aligned}
& \left\langle r^{2}\right\rangle^{88}-\left\langle r^{2}\right\rangle^{84}=-0.114(2) \mathrm{fm}^{2} \\
& \left\langle r^{2}\right\rangle^{88}-\left\langle r^{2}\right\rangle^{86}=-0.047(2) \mathrm{fm}^{2} \\
& \left\langle r^{2}\right\rangle^{88}-\left\langle r^{2}\right\rangle^{87}=-0.010(4) \mathrm{fm}^{2}
\end{aligned}
$$

we calculate the $F$ (slope) and $M$ (intercept) coefficients given in Table 3. The data of Table 3 allow us to do some further considerations about the sign of the volume isotope shifts. As expected, the $F$ coefficients result negative for both the 662 and $638 \mathrm{~nm}$ lines which involve a jump from an $s$ to an $p$ orbital. For a quantitative estimation of field shifts we can combine our $F$ values with the mean square radii of (1). For example, the $5 s 4 d^{3} D_{1}-5 p 4 d^{3} F_{2}$ transition exhibits a positive volume shift $F S^{88-86}=19$ (3) MHz, in spite of the negative $F$ value; this is a well known effect due to the closure of the neutron shell $N=50$ in ${ }^{88} \mathrm{Sr}$.

\section{Conclusions}

The present work reports hyperfine structure and isotope shifts in strontium. In particular we have investigated transitions involving two-excited electrons which are of relevant interest to study configuration mixing effects.

The specific mass and volume isotope effects are analyzed on the basis of the mean square charge radii reported in [5].

On the way to perform the present high resolution investigation we have also observed interesting optical pumping effects. For instance, for the transitions $5 s 5 p^{3} P_{1}-5 s 6 s^{3} S_{1}$ and $5 s 4 d^{3} D_{1}-5 p 4 d^{3} F_{2}$ the relative intensities of the hyperfine components appeared dramatically altered with respect to the even isotope peaks and strongly dependent on several experimental parameters (discharge pressure and laser intensity). Further investigations to better understand such phenomena are in progress in our laboratory also by using different spectroscopic techniques.

The authors are grateful to Prof. M. Inguscio for his encouragement and stimulating discussions during this work.

\section{References}

1. Aspect, A., Bauche, J., Fonseca, A.L.A., Grangier, P., Roger, G.: J. Phys. B 17, 1761 (1984)

2. Bender, D., Brand, H., Pfeufer, V.: Z. Phys. A - Atoms and Nuclei 318, 291 (1984)

3. Grundevik, P., Gustavsson, M., Lindgren, I, Olsson, G., Olsson, T., Rosén, A.: Z. Phys. A - Atoms and Nuclei 311, 143 (1983)

4. Godefroid, M., Vaeck, N., Hansen, J.E.: Numerical determina- 
tion of the electronic structure of atoms, diatomic and polyatomic molecules. p. 269. Dordrecht: Kluwer Academic Publishers 1989

5. Martin, A.G., Dutta, S.B., Rogers, W.F., Clark, D.L.: Phys. Rev. C 34, $1120(1986)$

6. Gianfrani, L., Monda, O., Sasso, A., Schisano, M.I., Tino, G.M., Inguscio, M.: Opt. Commun. 83, 300 (1991)

7. Inguscio, M.: J. Phys. (Paris) C 7, 44, 217 (1983)
8. Kopfermann, H.: In: Nuclear moments. New York: Academic Press 1958

9. Aspect, A., Bauche, J., Godefroid, M., Grangier, P., Hansen, J.E., Vaeck, N.: J. Phys: B 24, 4077 (1991)

10. Foot, C.J., Baird, P.E.G., Boshier, M.G., Stacey, D.N., Woodgate, G.K.: Opt. Commun. 50, 199 (1984)

11. Bauche, J., Champeau, R.J.: Adv. Atom. Molec. Phys. 12, 39 (1976) 